\title{
Antioxidant activity of the «litar» material
}

\begin{abstract}
The antioxidant status of the body is a very important indicator of health. It depends on the ability of body fluids to suppress free radicals. Therefore, the study of methods for analyzing body fluids to determine the total antioxidant capacity (TAC) and total antioxidant activity (CAOA) of the body are very important for medical practice. The composition of urine reflects changes in the body, diet and the content of polyphenol metabolites. At the same time, urine collection is a non-invasive method of analysis, which simplifies the determination of the antioxidant status of the body. The purpose of this work is to describe a non-invasive method for determining the antioxidant status of the body by the total antioxidant activity of urine using potentiometric coulometry on the example of "LitAR," a nanosized composite implantation material, approved for use in medical practice.
\end{abstract}

Keywords: the total antioxidant activity, antioxidant status of the body, total antioxidant activity of blood, saliva and urine, human oxidativestress biomarkers, nanoscale composite "LitAr», urine, coulonometric analysis metod
Volume 7 Issue 3 - 202 I

\author{
Petrov Sergey,' Lapin Anatoli,, Litvinov \\ Sergey ${ }^{3}$ \\ 'Department of Health and Safety, Moscow State Pedagogical \\ University, Russia \\ ${ }^{2}$ Department of Water Resources and Aquaculture, Kazan State \\ Energy University, Russia \\ ${ }^{3}$ Department of Natural Sciences, Reawis Medical University, \\ Russia
}

Correspondence: Petrov Sergey, Department of health and safety, Moscow State Pedagogical University, Russia, Tel+790350787 46, Email svpetrov47@mail.ru

Received: April 29, 2021 | Published: May 31, 2021

\section{Introduction}

To characterize antioxidant substances, the concept of "total antioxidant capacity" (TAC) was introduced. ${ }^{1-3}$ Oxidative stress is an imbalance between Reactive Oxygen Species (ROS) and human antioxidant defense. This risk factor arises under the influence of xenobiotics and endogenous processes. To assess the antioxidant status in vivo, it is necessary to take into account the synergistic interactions between antioxidants. Saliva analysis depends on oral hygiene and dental health. Urine samples are obtained non-invasively and this simplifies the body antioxidant status study. The composition of urine reflects changes in the body, diet and the content of polyphenol metabolites. ${ }^{4}$ To assess the ability of liquids to inhibit free radical reactions, we will use the term CAOA - "cumulative antioxidant activity" CAOA can be one of the criteria for monitoring of individualized therapy and evaluating the treatment of a pathological process, in the development of which processes of peroxidation can be noticed. ${ }^{5}$ When testing the effect of medicinal substances on urine CAOA, numerous data on the effect of food and beverages on its level have been accumulated. Walnuts and cocoa increased urine TAC 6 hours after taking. ${ }^{6-8} \mathrm{~A}$ decrease in urine TAC level was found after drinking of blackberry juice. ${ }^{9}$ But it was increased after eating spinach, strawberries ${ }^{10}$ cherries $^{11}$ and drinking drinks based on a mixture of apple, grape, blueberry and pomegranate juices and grape skins, grape seeds and green tea extracts as well as while eating a fatty meal. ${ }^{12} \mathrm{~A}$ drink based on a mixture of pineapple, blackcurrant and plum juice did not affect both urinary TAC and plasma TAC..$^{12}$ Drinking of grape juice during 5 days increased urinary TAC. ${ }^{13} \mathrm{An}$ increase in TAC was found when vitamin $\mathrm{C}(870 \mathrm{mg} / 1)$ was added to tomato juice. ${ }^{14}$ After a single or 2-week drinking of green or black coffee, the urine TAC level did not change. ${ }^{16,17}$ The purpose of this work is to describe a noninvasive method of determining the antioxidant status of the body by the total antioxidant activity of urine using potentiometric coulometry on the example of "Litar," an implantation material - a nanosized composite which is approved for use in medical practice.

\section{Materials and research methods}

"LitAr" is a material (Figure 1) and is a mixture of biopolymer (collagen or calcium-sodium alginate) and calcium hydroxophosphate, also called hydroxylapatite, hydroxyapatite or simply apatite. Figure 2 shows:
I. High degree of components structural integration, which favorably distinguishes it from other composites of similar types;

II. Nanometric sizes (43-45nm), at that the porosity of the material is about $70 \%$.

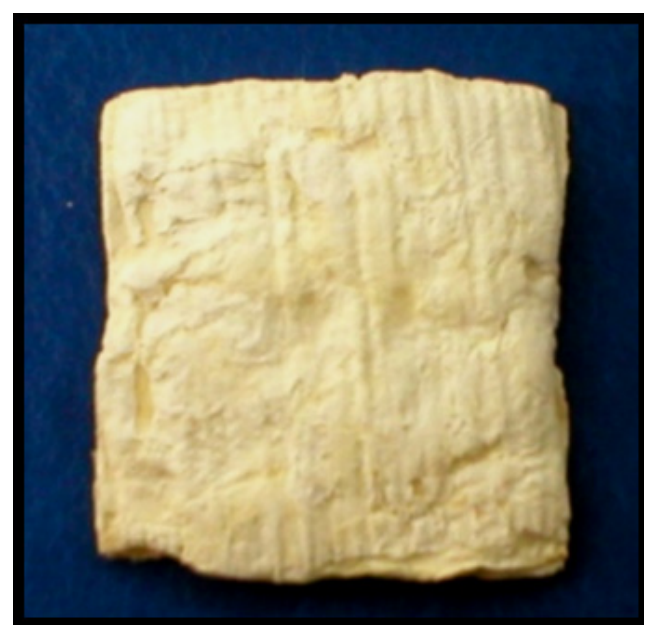

Figure I Material "litar" appearance.

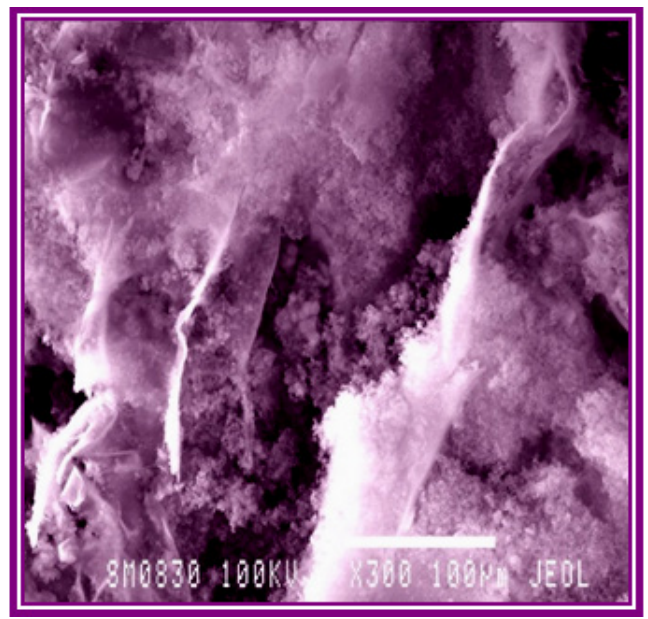

Figure 2 Material "litar" electron microscopy (magnification X 300). 
This is what provides the clinical effect: while the regeneration of conventional resorbable materials lasts months and years, it takes weeks for "LitAr." During the first 10-15 days, the implanted material is bi-transformed and gradually turned into the type of tissue (bone, cartilaginous or parenchymal) that was before the disease in the defective area of human tissue. ${ }^{15,18}$ The material can be administered either orally or by means of injection. When injected, the expected result is reliably predicted. Oral administration outcome is more difficult to predict if there are gastrointestinal, diabetic and vascular problems.

The study involved 3 participants: 2 men aged 67 and 75 years old and a woman 25 years old. They were practically healthy people, mainly of mental labor, who have not been sick for 3 months. The study was carried out under the ethical principles of the Helsinki Declaration of the World Medical Association. Control samples of morning urine were collected on an empty stomach at 7o'clock according to the Nechiporenko's method, after which "LitAr" $(0.5 \mathrm{~g})$ was taken. Urine samples were also collected on an empty stomach at 8 o'clock. Samples were analyzed without processing within an hour after collection. Evening urine samples were collected at 8p.m. or 9p.m. after taking "LitAr" $(0.5 \mathrm{~g})$ at $7 \mathrm{p} . \mathrm{m}$., at that they were stored in a refrigerator at $+6^{\circ} \mathrm{C}$ before analysis. A total of 30 urine samples with CAOA ranging from 285 to $1647 \mathrm{mg}$ rutin $(\mathrm{Ru})$ per $1 \mathrm{dm}^{3}$ (liter) were examined. The study results were determined by the effects of synergism (increase in CAOA) and antagonism (decrease in CAOA). CAOA indicators of evening urine (CAOA evening) taken at 8 p.m. relative to the control levels (CAOAcontr.) of morning urine samples taken at 7a.m. on an empty stomach according to the Nechiporenko method (Figure 1) were determined in relative percentage (\%rel.) according to the following formula: $\mathrm{CAOA}=100$ (CAOAevening. - CAOAcontr.): CAOAontr. The hydrogen ions $(\mathrm{pH})$ and the redox potential (Eh) activity indicators were measured on the I-160MI laboratory ionometer.

The samples CAOA levels were determined according to our certified method of the coulometric analysis by using electro generated bromine radicals done on an automated, certified, serial coulometer "Expert-006-Antioxidants" made by "Econix-Experts Ltd" (Moscow) ${ }^{19}$ The determination of CAOA levels was carried out in terms of a standard sample Ru level in mg. per $1 \mathrm{dm}^{3}$ of urine samples without conservation. The device was calibrated with an alcohol solution of the Russian standard sample (RSS) of $\mathrm{Ru}^{20}$ prepared according to the current State Pharmacopoeia of the $\mathrm{XI}^{\text {th }}$ edition. The obtained results statistical processing was carried out through a modal value (mode) of 10 determinations, ${ }^{21}$ the relative error in determining the $\mathrm{CAOA}$ of the whole urine (E rel.) was within 0.8-1.5\%. Oxidized samples (CAOoxid.) synengism and antagonism effects (CAOA) indicators in relation to the initial urine samples levels (CAOAorigin.) were determined in \% rel. according to the formula: $\mathrm{CAOA}=100$ (CAOAacid. - $\mathrm{CAOA}$ origin): $\mathrm{CAOA}$ origin. The urine samples were oxidized with $3 \%$ medical hydrogen peroxide (HP) produced by Iodine Technologies and Marketing Ltd. (Moscow, Russia) at a ratio of 1: 1 by volume. An increase in CAOA levels is not always a favorable sign. An increase in the CAOA level may indicate of the oxidative stress developing, accompanied with the mobilization of the antioxidant system. Uric acid (the main antioxidant of blood plasma which contribution is about $50 \%$ of CAOA) ${ }^{22}$ level increasing causes CAOA level increasing, and as in the case of renal failure with uremia, for example, causes oxidative damage to proteins and lipids and decreases the ascorbic acid concentration. ${ }^{23,24}$ Both the release of molecules of average weight $t^{25}$ or cell contents during necrosis and increase in the blood bilirubin level are also accompanied with CAOA level increase.

The study of HIV-infected patients CAOA levels done by us showed that the CAOA index of whole blood, which is significantly lower than in healthy people with minimal levels in the early stages of the disease is a more informative indicator of HIV-infected patients CAOA level, at that sick women CAOA blood level is reliably lower than that of men. ${ }^{26}$ Taking of 0.5 gr. of composite "LitAr" twice a day by a patient ( 67 years old) at $7 \mathrm{a} . \mathrm{m}$. and $7 \mathrm{p} . \mathrm{m}$. caused the effects of synergism (increase in CAOA) and antagonism (decrease in CAOA). Evening urine CAOA indicators (CAOAevening) taken at 8 p.m. in relation to the control levels (CAOAcontr.) of morning urine samples taken at 7 a.m. on an empty stomach according to the Nechiporenko method (Figure 3) in\% rel. are shown in Figure 3. The effects of synergism (increase in CAOA) and antagonism (decrease in CAOA) were also revealed when a patient (a 25-year-old woman) took the composite "LitAr" $(0.5 \mathrm{~g})$ twice a day at $7 \mathrm{a} . \mathrm{m}$. and $7 \mathrm{p} . \mathrm{m}$. CAOA of evening urine indicators (CAOAevening) taken at 8 p.m. in relation to the control values (CAOAcontr.) of morning urine samples taken at 7 a.m. on an empty stomach according to the Nechiporenko method in $\%$ rel. is shown in Figure 4.

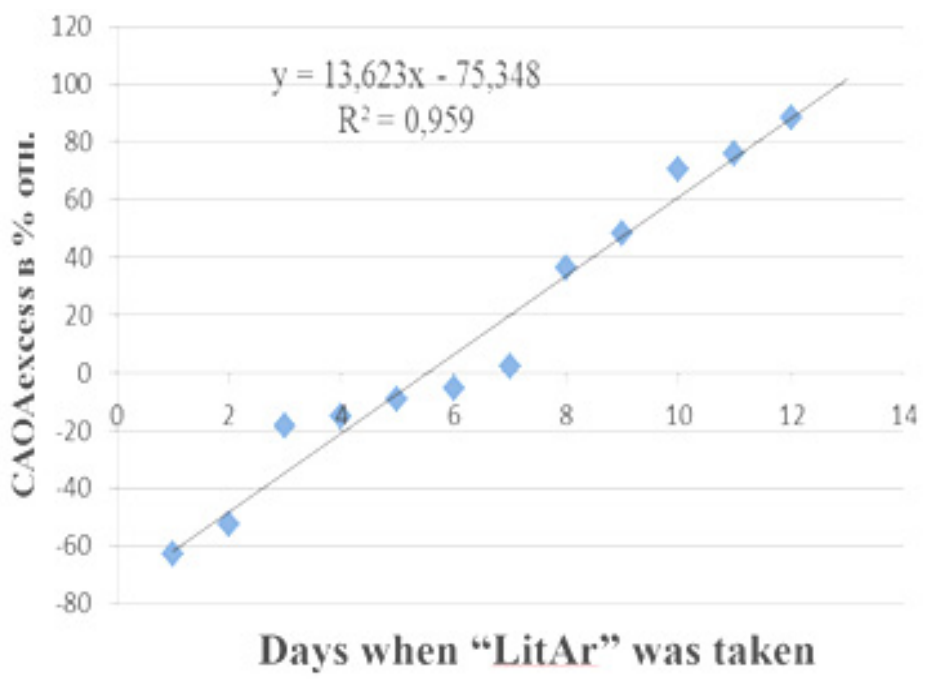

Figure 3 Increase and decrease in the cumulative antioxidant activity (CAOA) of evening urine indicators (CAOAevening) in relation to the control values (CAOAcontr.) of morning urine samples taken according to the Nechiporenko method in relative percentages during the period of taking the "litar" composite. 


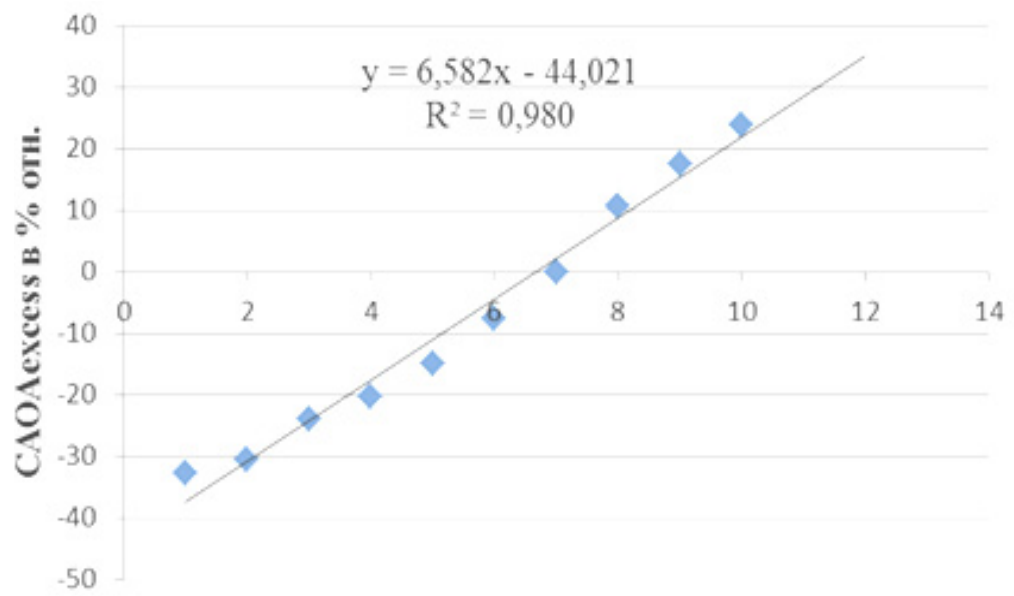

Days when "LitAr" was taken

Figure 4 Increase and decrease in the cumulative antioxidant activity (CAOA) of evening urine indicators (CAOAevening) in relation to the control values (CAOAcontr.) of the woman's morning urine samples taken by the Nechiporenko method in relative percentages during the period of taking the "litar" composite.

\section{Conclusion}

Human biofluids CAOA studies have both diagnostic and prognostic significance. They are becoming popular and may become standard laboratory tests. Human urine (TAC) studies have shown that these markers can be useful in assessing the antioxidant status of the body under the influence of diet, diseases and environmental conditions of human life. The effects of synergism (increase in total antioxidant activity) and antagonism (decrease in total antioxidant activity) of the relative levels of evening urine in the relation to the control values of morning urine taken on an empty stomach were revealed after patients' taking of $0.5 \mathrm{~g}$. of the "LitAr" composite in the morning and in the evening. For the first time, the effects of synergism and antagonism of the relative indicators of morning and evening urine samples that were oxidized with $3 \%$ medical hydrogen peroxide were revealed.

\section{Acknowledgments}

None.

\section{Conflicts of interest}

The authors declare that there is no conflict of interest.

\section{References}

1. Klebanov GI, Teselkin Yu O, Babenkova IV, et al. Antioxidant activity of blood serum. Bulletin of the RAMS. 1999;2:15-22.

2. Lapin AA, Viha GV. Indicator of total antioxidant activity of oral fluid is a non-invasive method of determining the antioxidant status of the body Butler messages [A1 ]. 201;22(12):8-45.

3. Ziobro A, Bartosz G. A comparison of the total antioxidant capacity of some human body fluids. Cell Mol Biol Lett. 2003;8(2):415-419.

4. Peluso I, Raguzzini A. Salivary and Urinary Total Antioxidant Capacity as Biomarkers of Oxidative Stress in Humans. Hindawi Publishing Corporation Pathology Research International.

5. Belyakov NA, Semesko SG. Antioxidant activity of human biological fluids. Methodology and clinical significance. Efferent therapy. 2005;11(1):5-21.

6. Hudthagosol C, Haddad E, Jongsuwat R. Antioxidant activity comparison of walnuts and fatty fish. Journal of the Medical Association of Thailand. 2012;95(6):179-188.
7. Pfundstein B, Haubner R, Würtele G, et al. Pilot walnut intervention study of urolithin bioavailability in human volunteers. Journal of Agricultural and Food Chemistry. 2014;62(42):10264-10273.

8. Roura E, Almajano MP, Bilbao MLM, et al. Human urine: epicatechin metabolites and antioxidant activity after cocoa beverage intake. Free Radical Research. 2007;41(8):943-949.

9. Hassimotto NMA, Pinto MDS, Lajolo FM. Antioxidant status in humans after consumption of blackberry (Rubus fruticosus L.) juices with and without defatted milk. Journal of Agricultural and Food Chemistry. 2008;56(24):11727-11733.

10. Cao G, Russell RM, Lischner N, et al. L. Serum antioxidant capacity is increased by consumption of strawberries, spinach, red wine or vitamin C in elderly women. Journal of Nutrition. 1998;128(12):2383-2390.

11. Garrido M, Paredes SD, Cubero J, et al. Jerte valley cherry-enriched diets improve nocturnal rest and increase 6-sulfatoxymelatonin and total antioxidant capacity in the urine of middle-aged and elderly humans. The Journals of Gerontology Series A: Biological Sciences and Medical Sciences. 2010;909-914.

12. Miglio C, Peluso I, Raguzzini A, et al. Fruit juice drinks prevent endogenous antioxidant response to high-fat meal ingestion. British Journal of Nutrition. 2014;111(2):294-300.

13. González-Flores D, Gamero E, Garrido M, et al. Urinary 6-sulfatoxymelatonin and total antioxidant capacity increase after the intake of a grape juice cv. Tempranillo stabilized with HHP. Food and Function. 2012;3(2.1):34-39.

14. Jacob K, Periago MJ, Böhm V, et al. Influence of lycopene and vitamin $\mathrm{C}$ from tomato juice on biomarkers of oxidative stress and inflammation. British Journal of Nutrition. 2008;99(1):137-146.

15. Petrov S, Valyaev A, Valiev A. Application of special nanomaterials for substituting bone tissue defects. Int $J$ Biosen Bioelectron. 2019;5(6):185-186.

16. Ziobro A, Bartosz G. A comparison of the total antioxidant capacity of some human body fluid. Cellular and Molecular Biology Letters. $2003 ; 8(2): 415-419$.

17. Revuelta-Iniesta R, Al-Dujaili EAS. Consumption of green coffee reduces blood pressure and body composition by influencing 11 $\beta$-HSD1 enzyme activity in healthy individuals: a pilot crossover study using green and black coffee. BioMed Research International. 2014.

18. Litvinov SD. The cytoactive biopolyver-salt base composite material ("LitAr") with nanosized crystals. Sheffield (GB). News of science and education; 2014;13:32-50. 
19. Zelenkov VN, Lapin AA. MVI-001-44538054-07. Total antioxidant activity. Procedure for performing measurements on a coulometric analyzer. All-Russian Research Institute of Vegetable Production; 2013:19.

20. A routine is a standard pattern. Specifications. TU 9369-141-0486824407.

21. Ezepov D. Fashion in statistics.2019.

22. Young IS. Measurement of total antioxidant capacity. J Clin Pathol. 2001;54(5):339.

23. Annuk M, Fellstrom B, Akerblom O, et al. Oxidative stress markers in pre-uremic patients. Clin Nephrol. 2001;56(4):308-314.
24. Bergesio F, Monzani G, Ciuti R, et al. Total antioxidant capacity (TAC): is it an effective method to evaluate the oxidative stress in uraemia? $J$ Biolumin Chemilumin. 1998;13(5):15-19.

25. Farkhutdinov RR, Likhovskikh VA. Chemiluminescent methods for the study of free radical oxidation in biology and medicine. BGMI Ufa. 1995:110.

26. Lapin AA, Mustafin IG, Borisenkov MF, et al. Coulometric Estimation of Blood Antioxidant Capacity at Aids Patients. Functional foods for chronic diseases. In: Danik M Martirosyan, editor. Texas, USA. 2009;4:76-85. 